Article

\title{
Clustering and Auction-Based Power Allocation Algorithm for Energy Efficiency Maximization in Multi-Cell Multi-Carrier NOMA Networks
}

\author{
Abuzar B. M. Adam, Xiaoyu Wan and Zhengqiang Wang *(1) \\ School of Communication and Information Engineering, Chongqing University of Posts \\ and Telecommunications, Chongqing 400065, China; abuzar@stu.xmu.edu.cn (A.B.M.A.); \\ wanxy@cqupt.edu.cn (X.W.) \\ * Correspondence: wangzq@cqupt.edu.cn; Tel.: +86-151-2300-9069
}

Received: 30 October 2019; Accepted: 19 November 2019; Published: 21 November 2019

\begin{abstract}
In this paper, we investigate the energy efficiency (EE) maximization in multi-cell multi-carrier non-orthogonal multiple access (MCMC-NOMA) networks. To achieve this goal, an optimization problem is formulated then the solution is divided into two parts. First, we investigate the inter-cell interference mitigation and then we propose an auction-based non-cooperative game for power allocation for base stations. Finally, to guarantee the rate requirements for users, power is allocated fairly to users. The simulation results show that the proposed scheme has the best performance compared with the existing NOMA-based fractional transmit power allocation (FTPA) and the conventional orthogonal frequency division multiple access (OFDMA).
\end{abstract}

Keywords: auction-based game; clustering; energy efficiency; multi-cell and multi-carrier; non-orthogonal multiple access (NOMA)

\section{Introduction}

The upcoming fifth-generation $(5 \mathrm{G})$ wireless communication system is expected to provide quality of service that fulfills the massive requirements of high data rates and the tremendous need for high connectivity and wireless data service. In such systems, excessive power consumption is inevitable and dealing with that dilemma is critical for mobile service operators. Currently, techniques such as orthogonal frequency division multiple access (OFDMA) have been exploited in the fourth generation $(4 \mathrm{G})$ wireless systems. In OFDMA, the frequency band is divided into orthogonal subchannels and each subchannel is assigned to one user at most [1].

As a promising technology, non-orthogonal multiple access (NOMA) is introduced for future wireless communication [2]. Unlike OFDMA, NOMA serves more than one user in a single subcarrier in a power domain. Consequently, the inter-user interference is inevitable, to deal with this problem; the successive interference cancelation (SIC) is employed at the receiver to decode the required information [3].

Since energy efficiency (EE) is a crucial measure of $5 \mathrm{G}$, more studies have been investigating energy saving by designing efficient power allocation for different NOMA systems. In Reference [4], the authors introduced an energy efficient power allocation for a multi-carrier NOMA (MC-NOMA) system, with the proportional rate constraints to guarantee fairness among users. The authors of Reference [5] put fairness as a priority in designing an energy efficient power allocation scheme for NOMA in ultra-dense heterogeneous networks. The study in Reference [6] considered designing the power allocation algorithm under imperfect channel state information. In Reference [7], Song et al. proposed a joint resource allocation algorithm in hybrid MC-NOMA to achieve spectrum efficiency (SE) 
and EE, where an arbitrary number of users can be multiplexed on the same subcarrier. In Reference [8], subcarrier and power are allocated jointly, using a time-efficient allocation algorithm. The proposed resource allocation framework was designed to maximize the sum rate in three main steps. In the first step, the problem was relaxed by assuming all subcarriers were available to use for each user. In the second step, subcarriers were allocated to users and in the third step, a centralized power allocation was introduced to allocate power to the users. Energy efficient resource allocation in multi-carrier scenario was investigated in Reference [9], the authors initially used matching theory to allocate users to subchannels. Then, the EE maximization problem was divided into subproblems which was solved by using penalty function method. Finally, power and subchannels were jointly allocated.

As an efficient and powerful method in strategic planning, game theory has been widely employed in resource allocation problems for wireless networks. In Liu et al. [10], a non-cooperative super-modular game was designed for subchannel matching to improve energy efficiency, the power was allocated equally for each subchannel and the game utility function was designed with the aim of maximizing user's EE, based on that, subchannel matching was designed. The auction-mechanism is a part of game theory, which has played a significant role in resource allocation. The authors of Reference [11] first separated the resource of the 5G network and then they designed a hierarchical combinatorial auction mechanism framework for resource allocation. The authors of Reference [12], introduced an auction-based resource allocation for software-defined networks. The auction-based power allocation game between users in NOMA network was addressed by Lamba et al. [13]. The base station (BS) played the auctioneer while users are the bidders. Simulation results have shown the effectiveness and outperformed other benchmark algorithms. Table 1 illustrates the comparison of different related works.

Table 1. Comparison of different related works.

\begin{tabular}{|c|c|c|c|c|c|c|c|c|}
\hline Study & [4] & [6] & [7] & [8] & [10] & [13] & [3] & This Study \\
\hline User Association & No & No & Yes & Yes & Yes & No & Yes & Yes \\
\hline $\begin{array}{l}\text { Interference } \\
\text { Management }\end{array}$ & No & No & No & No & No & No & No & Yes \\
\hline Network & $\mathrm{MC}$ & HetNet & $\mathrm{MC}$ & $\mathrm{MC}$ & $\mathrm{MC}$ & SC & MCMC & MCMC \\
\hline Topology & NOMA & NOMA & NOMA & NOMA & NOMA & NOMA & NOMA & NOMA \\
\hline Approach & $\mathrm{O}$ & $\mathrm{O}$ & $\mathrm{O}$ & $\mathrm{O}$ & $\mathrm{O} / \mathrm{GT}$ & GT & $\mathrm{O}$ & $\mathrm{O} / \mathrm{GT}$ \\
\hline
\end{tabular}

(O): Optimization, (GT): Game Theory, (ML): Machine Learning.

In this paper, we address the energy efficiency of MCMC-NOMA based networks with the constraints of rate requirements for users. From the above literature, different NOMA topologies have given considerable attention in academic research. However, few studies focus on the energy efficiency of MCMC-NOMA networks. The authors of Reference [14] have investigated energy minimization via determination of the optimal transmit power of the BS, which can achieve the optimal operating load of the cell. Hence, the motivations of this paper can be concluded as follows:

- Energy efficiency in MCMC-NOMA is not well studied and that gives more motivation to contribute on investigating this problem.

- Since such a topology entails inter-cell interference, as a consequence of inter-cell interference mitigation, the energy efficiency performance of the network can be significantly improved and that helps in the full exploitation of the potentials of NOMA. It is desirable to design a system that mitigates the inter-cell interference prior to the final power allocation.

Based on these motivations, the contributions of this paper are:

- The inter-cell interference mitigation is designed using $k$-means graph-based clustering. Thus, the network topology is divided into isolated interference regions. 
- Based on the aforementioned aspects of game theory, we consider auction mechanism to design a distributed power allocation scheme.

Finally, the simulation results demonstrate that the proposed algorithm outperforms the existing NOMA-based fractional transmit power allocation (NOMA-FTPA) and conventional OFDMA system in terms of energy efficiency.

\section{System Model and Problem Formulation}

\subsection{System Model}

We consider a downlink of MCMC-NOMA system, including $M$ cells each cell involving a single base station (BS) serving $N$ randomly distributed users through $K$ subcarriers. In this scenario, we assume that each BS and users are equipped with a single antenna. Simply, we denote $\mathcal{M}=\{1,2, \ldots ., M\}, \mathcal{N}=\{1,2, \ldots ., N\}, \mathcal{S}=\{1,2, \ldots, K\}$ as the set of cells, subcarriers set and users, respectively.

According to the NOMA protocol, all BSs simultaneously transmit a superposition signal to its users. For notational simplicity, we use $m, i, l$ to denote the user $i$ connecting to BS $m$ at $l$ subcarrier.

$$
y_{m, i, l}=H_{m, i, l} \chi_{m, l}+\eta_{m, i, l}+I_{m, i, l}^{\text {inter }},
$$

where $H_{m, i, l}=g_{m, i, l} d_{m, i, l}^{-\alpha}$ is the channel coefficient between BS $m$ and its associated user $i$ at subcarrier $l, g_{m, i, l}$ is the Rayleigh fading channel gain, $d_{m, i, l}$ is the distance between the BS and its associated user and $\alpha$ is path loss exponent; $\chi_{m, l}$ is the transmitted signal of the BS $m$ to its associated user through subcarrier $l ; \eta_{m, i l}$ is the additive white Gaussian noise (AWGN) with zero mean and variance $\delta_{m, i, l}^{2}$; $I_{m, i, l}^{\text {inter }}$ is the inter-cell interference received by user $i$ from other users in the same subcarrier. The normalized channel gain for user $i$ can be denoted $h_{m, i, l}=\frac{\left|H_{m, i, l}\right|^{2}}{\delta_{m, i, l}^{2}+I_{m, i, l}^{\text {inter }}}$.

According to the principle of NOMA, successive interference cancellation (SIC) can be employed at some of the users with better channel conditions, while the signal of the users with better channel conditions are first decoded and subtracted, then the information for the user with worse channel conditions is decoded. Without loss of generality, we assume users are ordered according to their normalized gains for optimal decoding as $h_{m, 1, l} \geq h_{m, 2, l} \geq \ldots . \geq h_{m, n, l}$, then signal-to-interference-plus-noise-ratio (SINR) for user $i$ with the SIC at receiver can be given as

$$
\operatorname{SINR}_{m, i, l}=\frac{v_{m, i, l} Y_{m, i, l} h_{m, i, l} p_{m, i, l}}{1+\sum_{j=i+1} v_{m, i} Y_{i, l} h_{m, i, l} p_{m, j, l}+\sum_{n \neq m} h_{n, i, l} P_{n, l}},
$$

where $v_{m, i} \in[0,1]$ and $\mathrm{Y}_{i, l} \in[0,1]$ respectively are the BS and sub-channel assignment indexes, when the value is 1 , the user is associated with the $m$-th BS through the $l$-th subchannel, $l \in S$ is the sub-channel, the term $\sum_{n \neq m} h_{n, i, l} P_{n, l}$ represents the aggregated inter-cell interference at the $i$-th user from other users on the same channel served by other BSs in which $P_{n, l}$ is the total power consumption of the of the $n$-th BS on the same sub-channel given as

$$
P_{n, l}=\sum_{n=1, n \neq m}^{\mathcal{N}} v_{n, i, l} Y_{n, i, l} P_{n, i, l}
$$

Then, the data rate of the user $i$ can be expressed as

$$
R_{m, i, l}=\beta \log _{2}\left(1+S I N R_{m, i, l}\right),
$$


where $\beta$ is the subcarrier's bandwidth and the sum rate of the network can be written as

$$
R=\sum_{m=1}^{\mathcal{M}} \sum_{i=1}^{\mathcal{N}} \sum_{l \in S} R_{m, i, l}
$$

Finally, the energy efficiency of the MCMC-NOMA is given by

$$
E E_{t}=\frac{R}{P_{T}}
$$

where $P_{T}=\sum_{m=1}^{\mathcal{M}}\left(P_{m}+P_{m}^{c}\right)$ is the total power, $P_{m}$ and $P_{m}^{c}$ are respectively represent the transmit power and circuit power consumption of BS $m$. We assume each user is associated with one BS and cannot be assigned with more than one subcarrier.

\subsection{Problem Formulation}

In this part, we to aim to find the energy efficiency of network, the EE problem is formulated with maximum transmit power constraints and quality of service (QoS) for users. Hence, the EE optimization problem can be formulated as

$$
\begin{aligned}
\max _{v, Y, p} E E_{t}= & \frac{R}{P_{T}} \\
\text { subject to } \quad & \sum_{i=1}^{\mathcal{N}} \sum_{l \in \mathcal{S}} v_{m, i, l} Y_{m, i, l} p_{m, i, l} \leq P_{m}, \forall m \in \mathcal{M} \\
& p_{m, i, l} \geq 0, \forall m \in M, i \in \mathcal{N}, l \in \mathcal{S} \\
& \sum_{l \in \mathcal{S}} v_{m, i, l} Y_{m, i, l} R_{m, i, l} \geq R_{m, i, l}^{t h}, \forall m \in \mathcal{M}, i \in \mathcal{N} \\
& \sum_{l \in \mathcal{S}} v_{m, i, l} Y_{m, i, l} \leq 1, \forall m \in \mathcal{M}, i \in \mathcal{N} .
\end{aligned}
$$

The first constraint $(7 \mathrm{~b})$ denotes the total transmit power constraint for all users served by BS $m$. (7c) denotes the user's power constraint. (7d) is the minimum data rate requirements for user. (7e) means that the user can be associated with one BS and assigned to one subcarrier at the time.

\section{Proposed Method}

In this part, we divide the proposed method into two parts, since MCMC-NOMA topology usually entails inter-cell interference, our first task is to mitigate the interference then introduce an optimal power allocation scheme.

\subsection{Interference Mitigation}

Clustering can effectively improve the management ability of the network to reduce the inter-cell interference. We use graph-based k-means [15] downlink clustering for interference elimination.

Let us redefine the aggregated inter-cell interference at the $i$-th user served by $m$-th BS from other users assigned to the same sub-channel and served by other BSs

$$
I_{m, i, l}^{\text {inter }}=\sum_{n=1, n \neq m}^{\mathcal{M}} v_{n, i, l} \mathrm{Y}_{n, i, l} p_{n, i, l} d_{n, i, l}^{-\alpha}
$$

To build up the potential interference relationship between the users in the different base stations, we construct an interference graph. We define the points representative of the downlink of each base station to its associated user. 
Let us define our graph as $\mathbb{G}=(\mathcal{V}, \mathbb{E})$, where $\mathcal{V}$ represents the vertices which are the communication devices and $\mathbb{E}$ represents the set of edges that correspond to the interference level received by the $i$-th user from other BSs, this interference level is calculated by (8) after the initial assignment of power.

The benefit of cluster is that no matter the complexity of the environment, we consider the relationship between users who interfere with each other. According to the strength of interference received by the user's and with setting up a specific threshold, the network is divided into numerous interference areas similar to Reference [16], which correspond to the coverage areas of each base station. For convenience, we will call interference areas as clusters. Once we finish cell association and since our proposed power allocation scheme increasingly allocates power and as a consequence that leads to increment in interference, nevertheless, any interference value above the threshold can be neglected. Consequently, the potentials of NOMA to manage the interference can be employed.

\subsection{Auction-Based Non-Cooperative Game}

After we have obtained user association by interference mitigation, then we need to allocate power for each user suitable to maximize the EE. We will use auction-based Non-cooperative game to allocate power to our clusters.

The auction mechanism involves several hierarchical models [11], Single-seller multiple-buyer hierarchical auction model is used in our case, we assume there's a central controller which can manage the resource allocation process. Hence, we introduce an auction-based non-cooperative game to allocate power efficiently. The result from the previous clustering and the interference mitigation process is used, each cluster consists of one base station, then the non-cooperative game is utilized to allocate the power to the clusters based on their submitted bids. Each cell is assumed to be selfish and always chooses a strategy to maximize its utility. In the following lines, we express the mathematical model of the game.

Utility Function

The power allocation non-cooperative game is denoted as

$$
\mathcal{G}=\mathcal{G}\{\aleph, \mathcal{B}, \mathcal{U}\}
$$

where:

$\aleph$ : set of bidders (clusters).

$\aleph=\{1,2, \ldots, \mathcal{C}\}$.

$\mathcal{B}_{c}$ : Auction space of each bidder $c \in \aleph$.

$\mathcal{U}_{c}: \mathcal{B} \equiv \prod_{c} \mathcal{B}_{c} \rightarrow P_{t}$ is the cluster's utility function defined by (10).

From the mathematical definition of energy efficiency, we need the balance the date and the power consumption. Therefore, in the proposed game, each cluster is competing to maximize its sum rate with the consideration of price for the power consumption. Consequently, the utility function of the cluster $c$ can be defined as:

$$
\mathcal{U}_{c}(t)=\mathcal{R}_{c}(t)-b_{c}(t) p_{c}(t),
$$

where $\mathcal{R}_{c}(t)$ is the sum rate of all users within the cluster $c, b_{c}(t)$ represents the submitted bid by the cluster and $P_{t}$ is the total offered resource for auctioning, $t$ is the iteration index. $p_{c}(t)$ is the fraction of power assigned to the cluster based on its submitted bid. To initiate the auction process, the clusters can obtain their portion equally at an initial bids $\left(b_{c}(0)\right)$ equal to the reserved price $\sigma$, thus,

$$
p_{c}(0)=P_{t} \frac{b_{c}(0) h_{c}}{\sum_{k=1}^{\mathcal{C}} b_{k}(0) h_{k}}
$$


when cluster c submits a new bid $b_{c}(t)$, the allocated power is updated as follows

$$
p_{c}(t+1)=P_{t} \frac{b_{\mathcal{c}}(t) p_{\mathcal{c}}(t)}{\sum_{k=1}^{\mathcal{C}} b_{k}(t) p_{k}(t)+\sigma},
$$

taking a derivative of (10) and after rearranging the terms, the bidding status can be updated as:

$$
b_{c}(t+1)=\frac{(\ln 2)^{-1} h_{c}}{1+h_{c} p_{c}(t+1)+\sum_{k=1, k \neq c}^{\mathcal{C}} h_{k} p_{k}(t+1)}
$$

where $h_{c}$ represents the aggregated gain of all $\mathcal{N}$ users within cluster $c$ and it can be calculated as:

$$
h_{c}=\sum_{i=1}^{\mathcal{N}} h_{i}
$$

$h_{k}$ represents the aggregated gains in other clusters. After power is allocated to the winner, he is kicked out of the auction. Total offered power is updated after deducting the winner's power. Then, a new round of auctioning starts. Auctioning resumes with new $P_{t}$ if the previous offered resource was not enough.

One of the main objectives of QoS is the fairness. To grant the fairness and quality of service for all users, fair power allocation (FPA) [5] scheme is adopted to see each user's power need properly attended then the user's rate is calculated to check whether rate constraint is fulfilled. Algorithm 1 explains the mechanism of our proposed power allocation scheme. Finally, Jain's fairness index [17] is adopted to measure the fairness of power allocation algorithm, the definition of this index is given as

$$
J=\frac{\left(\sum_{i=1}^{\mathcal{N}} R_{m, i, l}\right)^{2}}{\mathcal{N} \sum_{i=1}^{\mathcal{N}} R_{m, i, l}^{2}} .
$$

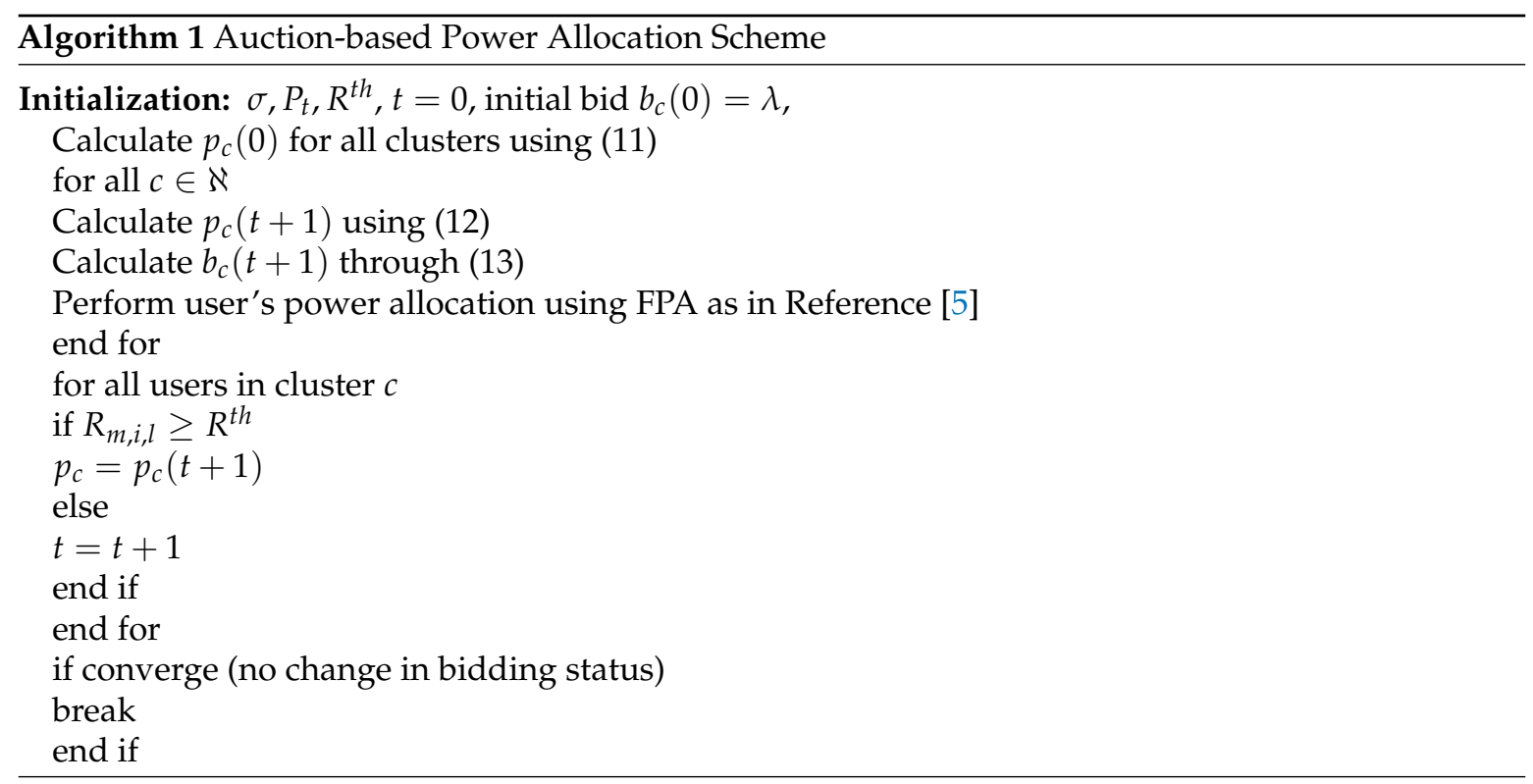




\section{Simulation Results}

In this section, we analyze the performance of the proposed power allocation scheme and the comparison with fractional transmit power allocation (FTPA) [18] benchmark algorithm for the NOMA and OFDMA algorithms in Reference [19]. Table 2 shows the simulation parameters.

Table 2. Simulation Parameters.

\begin{tabular}{cc}
\hline Parameter & Value \\
\hline Path loss exponent & 3.7 \\
Shadowing & Log-normal, standard deviation $8 \mathrm{~dB}$ \\
Fading & Rayleigh fading with variance 1 \\
Noise power spectral density & $-174 \mathrm{dBm} / \mathrm{Hz}$ \\
Subcarrier band & $180 \mathrm{kHz}$ \\
\hline
\end{tabular}

Firstly, the convergence of the algorithm is shown in Figure 1 for a total transmit power $P_{t}=20 \mathrm{dBW}$ and $10 \mathrm{BS}$ with 10 users for each BS, we can notice the algorithm converges in a few iterations.

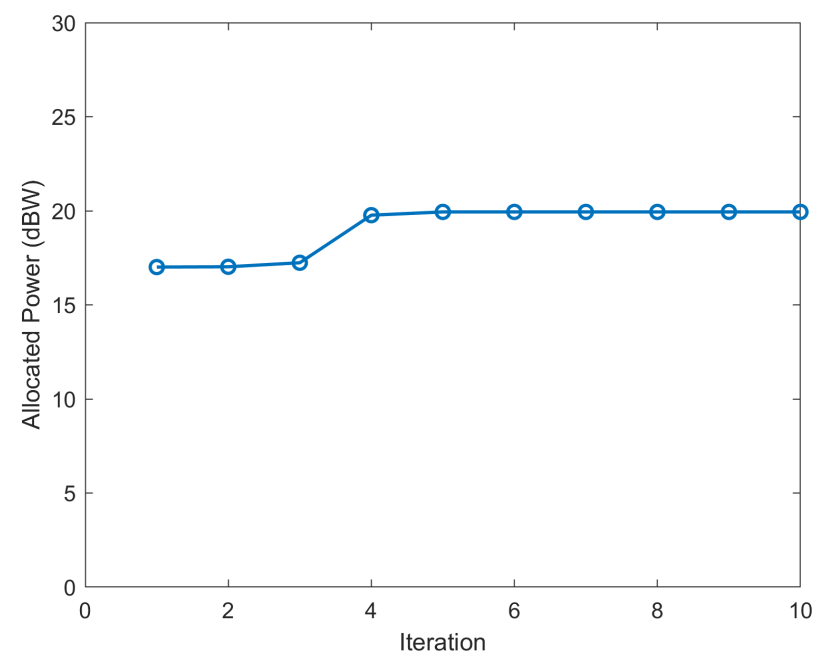

Figure 1. Convergence of the proposed power allocation scheme.

Figure 2 compares the performance of the proposed algorithm for different multi-carrier cases with the benchmark algorithms in terms of the transmit power consumption for different data rate values. From the figure, one can notice that the proposed algorithm outperforms NOMA-FTPA and OFDMA.

From Figure 3, it is easy to observe that the proposed power allocation scheme is the most energy efficient followed by NOMA-FTPA and OFDMA, the result in Figure 3 simulating 10 BSs with 10 users for each. Moreover, unlike the approaches of comparison, in the proposed method; EE increases with the transmit power.

In Figure 4, the performance of total sum rate is evaluated with the number of users varies from 10 to 60 per BS. We observe that the performance of the MCMC-NOMA proposed power allocation algorithm is much better than the NOMA-FTPA and OFDMA schemes. 


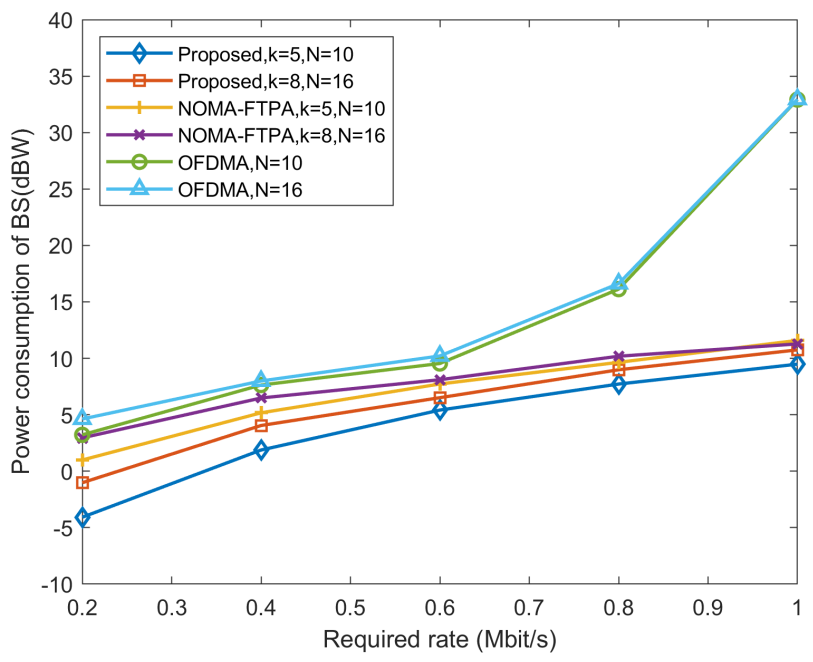

Figure 2. Power consumption of base station (BS) for different data rate requirements and different multi-carrier cases.

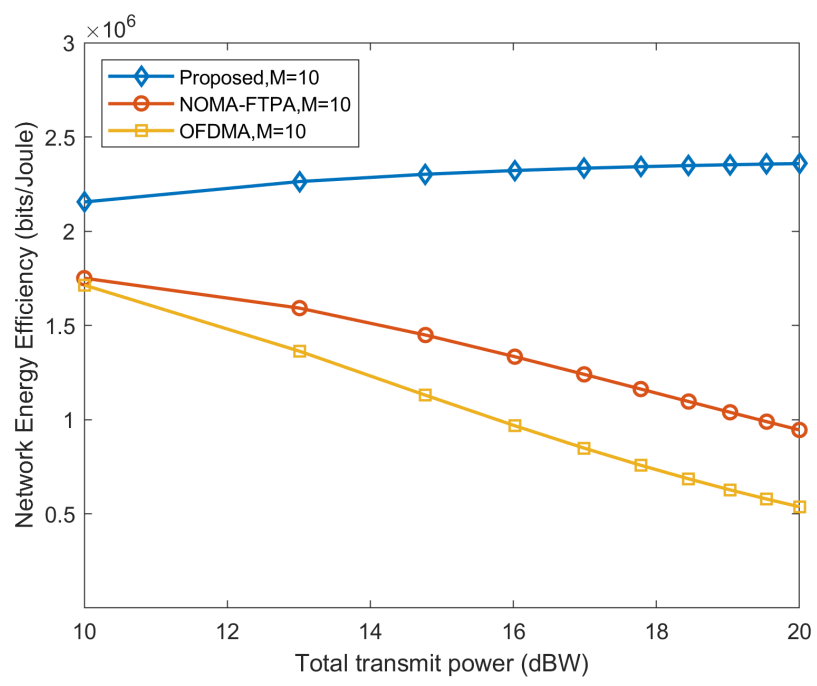

Figure 3. Network energy efficiency performance for different transmit power of cell.

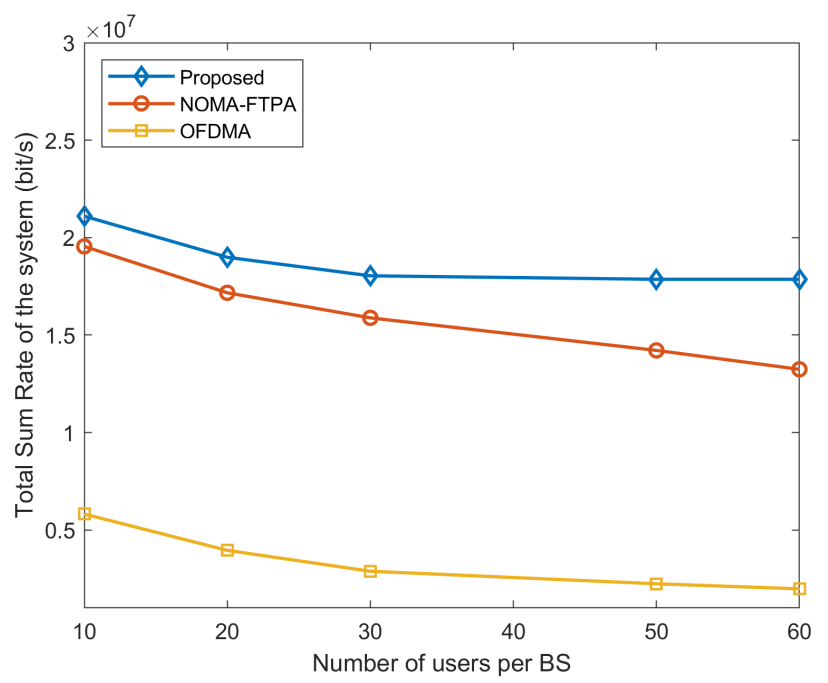

Figure 4. Total sum rate of the system versus different number of users per BS. 
Figure 5 shows the energy efficiency versus the number of users with the same constraints of Figure 4. Although both proposed methods and NOMA-FTPA have higher energy efficiency with a lower number of users (10 user per BS), yet energy efficiency drastically decreasing with the increasing number of users. The performance of the proposed method is $12.46 \%$ better than NOMA-FTPA, and we can notice this performance gap is almost remaining fixed as the number of users increases. We can observe that the performance gaps between NOMA schemes (proposed algorithm and FTPA) and OFDMA are higher when the number of users is decreasing. The performance gap between the proposed algorithm and OFDMA stands about $35.1 \%$ when the number of users is 10 per BS and dramatically deteriorating to $22.5 \%$ when the number of users is 60 per BS.

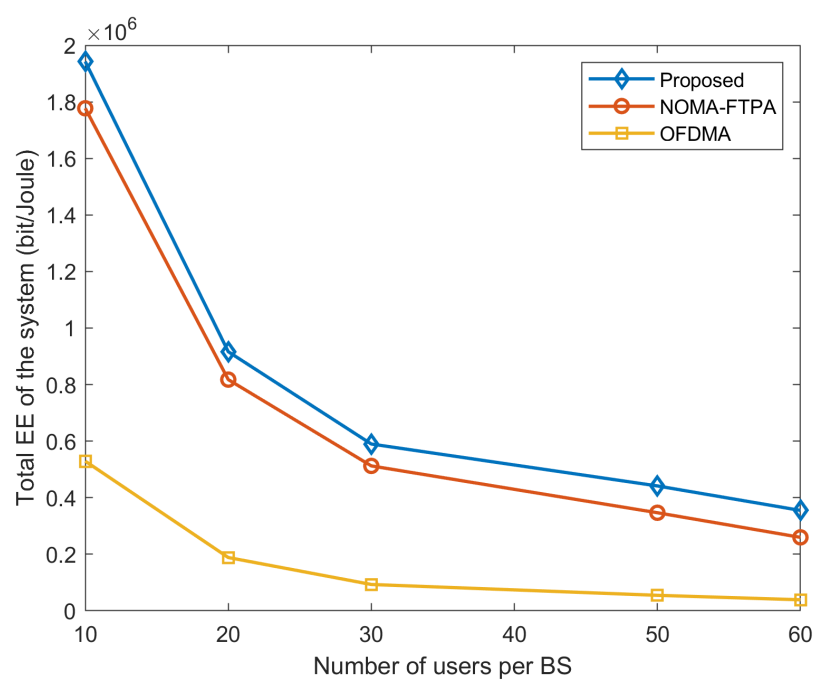

Figure 5. Total energy efficiency versus different number of users per BS.

Figure 6 shows the total energy efficiency versus $P_{c} /$ transmitpower ratio. According to the definition of energy efficiency, its value should decrease when the value of circuit power increases. However, the proposed MCMC-NOMA power allocation algorithm still has the best performance among the other compared algorithms.

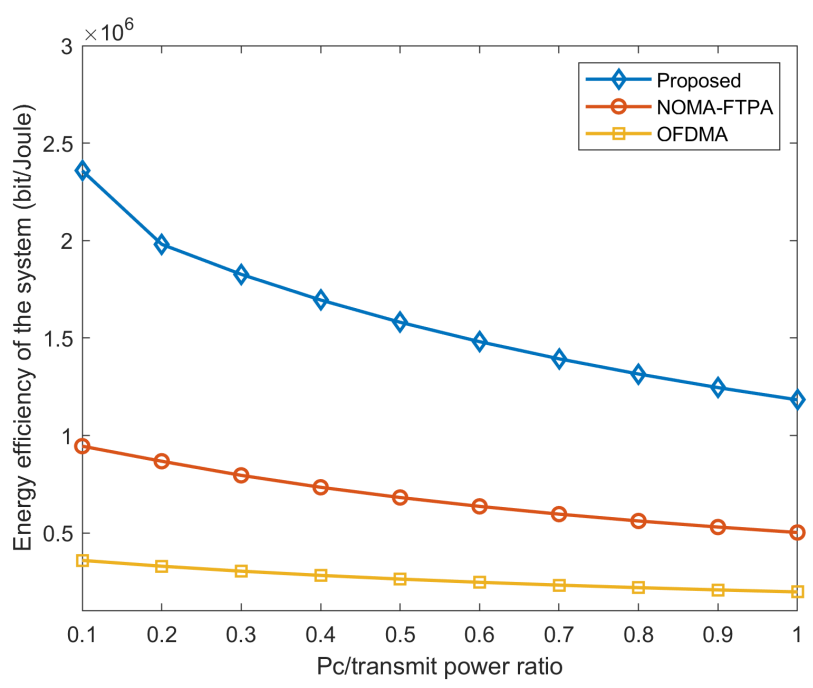

Figure 6. Energy efficiency of the system versus $P_{c} /$ transmit power ratio.

Regarding the fairness in power allocation, Figure 7 demonstrates a comparison between the three approaches considered in this study for fixed transmit power of $10 \mathrm{dBW}$ and different number of users. From the figure, one can observe the proposed algorithm outperforms the other compared 
approaches. When the number of users is 10 users, the fairness index measures $0.967731,0.9264876$ and 0.4450965 for the proposed method, NOMA-FTPA and OFDMA respectively but when the number of users is increasing, the fairness is deteriorating for both NOMA-FTPA and OFDMA while it is slight in the case of our proposed method.

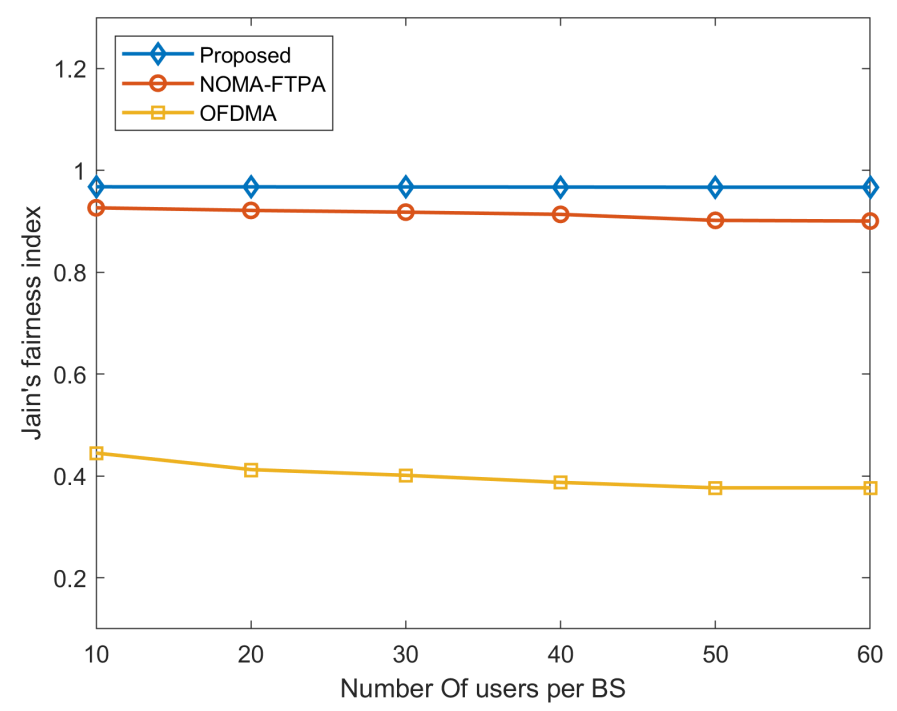

Figure 7. Comparison between different methods in term of fairness.

\section{Conclusions}

In this paper, we proposed an energy efficient power allocation algorithm aiming to maximize the energy efficiency of MCMC-NOMA networks based on the auction theory. First, we investigated interference mitigation using graph-based k-means clustering. Then, an auction-based power allocation scheme is proposed to allocate power to the BSs. Finally, the power is fairly allocated to users. Simulation results show that the proposed scheme converges with fast speed. Moreover, the overall performance of the proposed MCMC-NOMA algorithm has significantly improved the energy efficiency of the network and surpassed other existing schemes such as NOMA-FTPA and OFDMA.

Author Contributions: Z.W. conceived and designed the system model; X.W. analyzed the data and contributed the formal analysis; A.B.M.A. performed the simulation and wrote the paper.

Funding: This work was funded by the National Natural Science Foundation of P. R. China (No. 61701064), the Chongqing Natural Science Foundation (cstc2019jcyj-msxmX0264).

Conflicts of Interest: The authors declare no conflict of interest.

\section{Abbreviations}

The following abbreviations are used in this manuscript:

EE

MCMC-NOMA multicell multicarrier non-orthogonal multiple access

FTPA fractional transmit power allocation

OFDMA orthogonal frequency division multiple access

$5 \mathrm{G}$

fifth generation

SE

spectrum efficiency

BS base station

SINR signal to interference plus noise ratio

SIC successive interference cancellation

QoS quality of service

FPA fair power allocation 


\section{References}

1. Cui, J.; Liu, Y.; Ding, Z.; Fan, P.; Nallanathan, A. QoE-Based Resource Allocation for Multi-Cell NOMA Networks. IEEE Trans. Wirel. Commun. 2018, 17, 6160-6176. [CrossRef]

2. Fang, F.; Ding, Z.; Liang, W.; Zhang, H. Optimal Energy Efficient Power Allocation with User Fairness for Uplink MC-NOMA Systems. IEEE Wirel. Commun. Lett. 2019, 8, 1133-1136. [CrossRef]

3. Ni, D.; Hao, L.; Tran, Q.T.; Qian, X. Transmit Power Minimization for Downlink Multi-Cell Multi-Carrier NOMA Networks. IEEE Commun. Lett. 2018, 22, 2459-2462. [CrossRef]

4. Yuan, Z.; Li, X.; Lv, G. Energy Efficient Power Allocation for Multi-carrier Non-orthogonal Multiple Access (NOMA) Systems with Proportional Rate Constraints. In Proceedings of the 19th International Conference on Science and Technology of Automatic Control and Computer Engineering (STA), Chengdu, China, 15-17 March 2019; pp. 611-613.

5. Xiang, L.; Chen, H. Energy-Efficient and Fair Power Allocation Approach for NOMA in Ultra-Dense Heterogeneous Networks. In Proceedings of the International Conference on Cyber-Enabled Distributed Computing and Knowledge Discovery (CyberC), Nanjing, China, 12-14 October 2017; pp. 89-94.

6. Song, X.; Dong, L.; Wang, J.; Qin, L.; Han, X. Energy Efficient Power Allocation for Downlink NOMA Heterogeneous Networks With Imperfect CSI. IEEE Access 2019, 7, 39329-39340. [CrossRef]

7. Song, Z.; Ni, Q.; Sun, X. Spectrum and Energy Efficient Resource Allocation With QoS Requirements for Hybrid MC-NOMA 5G Systems. IEEE Access 2018, 6, 37055-37069. [CrossRef]

8. Fu, Y.; Salaun, L.; Sung, C.W.; Chen, C.S. Subcarrier and Power Allocation for the Downlink of Multicarrier NOMA Systems. IEEE Trans. Veh. Technol. 2018, 67, 11833-11847. [CrossRef]

9. Huang, K.; Wang, Z.; Zhang, H.; Fan, Z.; Wan, X.; Xu, Y. Energy efficient resource allocation algorithm in multi-carrier noma systems. In Proceedings of the IEEE 20th International Conference on High Performance Switching and Routing (HPSR), Xi'An, China, 26-29 May 2019; pp. 1-5.

10. Liu, G.; Wang, R.; Zhang, H.; Kang, W.; Tsiftsis, T.A.; Leung, V.C.M. Super-Modular Game-Based User Scheduling and Power Allocation for Energy-Efficient NOMA Network. IEEE Trans. Wirel. Commun. 2018, 17, 3877-3888. [CrossRef]

11. Zhu, K.; Hossain, E. Virtualization of 5G Cellular Networks as a Hierarchical Combinatorial Auction. IEEE Trans. Mob. Comput. 2016, 15, 2640-2654. [CrossRef]

12. D'Oro, S.; Galluccio, L.; Mertikopoulos, P.; Morabito, G.; Palazzo, S. Auction-based resource allocation in OpenFlow multi-tenant networks. Comput. Netw. 2017, 115, 29-41. [CrossRef]

13. Lamba, A.K.; Kumar, R.; Sharma, S. Auction-based power allocation for downlink non-orthogonal multiple access systems. Electron. Lett. 2019, 55, 420-422. [CrossRef]

14. Lei, L.; You, L.; Yang, Y.; Yuan, D.; Chatzinotas, S.; Ottersten, B. Power and Load Optimization in Interference-Coupled Non-Orthogonal Multiple Access Networks. In Proceedings of the 2018 IEEE Global Communications Conference (GLOBECOM), Abu Dhabi, UAE, 9-13 December 2018; pp. 1-6.

15. Galluccio, L.; Michel, O.; Comon, P.; Hero, A.O., III. Graph based k-means clustering. Signal Process. 2012, 92, 1970-1984. [CrossRef]

16. Jeon, H.; Koo, B.; Chae, C.; Park, S.; Lee, H. Game theory based hybrid frequency assignment with net filter discrimination constraints. ICT Express 2019, 5, 89-93. [CrossRef]

17. Jain, R.; Chiu, D.; Hawe, W. Quantitative Measure of Fairness and Discrimination for Resource Allocation in Shared Computer Systems; DEC Research Report TR-301; Digital Equipment Corporation: Maynard, MA, USA, 1984. [CrossRef]

18. Saito, Y.; Kishiyama, Y.; Benjebbour, A.; T Nakamura, A.L.; Higuchi, K. Non-Orthogonal Multiple Access (NOMA) for Cellular Future Radio Access. In Proceedings of the IEEE 77th Vehicular Technology Conference (VTC Spring), Dresden, Germany, 2-5 June 2013; pp. 1-5.

19. Yang, Z.; Xu, H.; Shi, J.; Pan, Y.; Li, Y. Power control and resource allocation for multi-cell ofdm networks. In Proceedings of the IEEE Conference on Computer Communications Workshops (INFOCOM WKSHPS), San Francisco, CA, USA, 10-14 April 2016; pp. 891-896.

(C) 2019 by the authors. Licensee MDPI, Basel, Switzerland. This article is an open access article distributed under the terms and conditions of the Creative Commons Attribution (CC BY) license (http:/ / creativecommons.org/licenses/by/4.0/). 